\title{
A Combined MMSE-ML Detection for a Spectrally Efficient non Orthogonal FDM Signal
}

\author{
Ioannis Kanaras $^{\dagger}$, Arsenia Chorti ${ }^{\dagger}$, M.R.D. Rodrigues ${ }^{\ddagger}$, and Izzat Darwazeh ${ }^{\dagger}$ \\ ${ }^{\dagger}$ Telecommunications Research Group, Dept. E\&EE, University College London \\ London, UK, Email: i.kanaras@ee.ucl.ac.uk, a.chorti@ee.ucl.ac.uk, i.darwazeh@ee.ucl.ac.uk \\ ${ }^{\ddagger}$ Instituto de Telecomunicações, Department of Computer Science, University of Porto \\ Porto, Portugal, Email: mrodrigues@dcc.fc.up.pt
}

\begin{abstract}
In this paper, we investigate the possibility of reliable and computationally efficient detection for spectrally efficient non-orthogonal Multiplexing (FDM) system, exhibiting varying levels of intercarrier interference. Optimum detection is based on the Maximum Likelihood (ML) principle. However, ML is impractical due to its computational complexity. On the other hand, linear detection techniques such as Zero Forcing (ZF) and Minimum Mean Square Error (MMSE) exhibit poor performance. Consequently, we explore the combination of MMSE estimation with ML estimation around a neighborhood of the MMSE estimate. We evaluate the performance of the different schemes in Additive White Gaussian Noise (AWGN), with reference to the number of FDM carriers and their frequency separation. The combined MMSE-ML scheme achieves a near optimum error performance with polynomial complexity for a small number of BPSK FDM carriers. For QPSK modulation the performance of the proposed system improves for a large number of ML comparisons. In all cases, the detectability of the FDM signal is bounded by the signal dimension and the carriers frequency distance.
\end{abstract}

\section{INTRODUCTION}

T He ever growing demand for broadband applications has been a key driver for research into spectrally efficient physical layer techniques that guarantee reliable transmission and reception of high data rate signals. Orthogonal Frequency Division Multiplexing (OFDM) prevails in the area of wired and wireless communications and is currently employed in diverse communication systems such as the Digital Subscriber Line (DSL), the Digital Audio and Video Broadcasting (DAB and DVB, respectively), 802.11a,g as well as the broadband wireless access networks (WiMAX).

Recently, Rodrigues and Darwazeh [1] as well as Xiong [2] introduced systems that occupy half the OFDM bandwidth. However, the spectral efficiency benefit is not fully exploited since these systems can only operate with one-dimensional modulation (e.g. BPSK or M-ASK). Moreover, they appear to be highly sensitive to wireless environments impairments such as frequency offsets, timing offsets, and frequency selective fading [3].

Additionally, Rodrigues and Darwazeh proposed a nonorthogonal Frequency Division Multiplexing (FDM) system [4]. This system exhibits higher spectral efficiency, at the expense of higher detection complexity. For such systems it was shown that ML detection is not computationally feasible
[4]. On the other hand, linear Zero Forcing (ZF) detection exhibited unsatisfactory error performance.

Consequently, to tackle the detection problem, we investigate the performance of alternative techniques. In particular, we consider the linear Minimum Mean Squared Error (MMSE) detection. In addition, stimulated by relevant research results in Multiple-Input Multiple-Output (MIMO) systems area [5], [6], and [7], we introduce and evaluate a combined MMSE ML scheme.

This paper is organised as follows: In sections II and III we describe the FDM system and alternative detection techniques. In section IV we provide simulation results concerning the error performance and the complexity of the methods. Finally, we summarize our main conclusions, outlining future research directions.

\section{FDM SYSTEM MODEL}

The original FDM system architecture is described in [4]. Initially, the input stream is split into $N$ parallel streams that modulate $N$ different carriers according to a modulation scheme of size $M$. The FDM carriers are separated in frequency by a distance $\Delta f$ that is only a fraction of the inverse of the FDM symbol period $T$. Consequently, the bandwidth of the transmitted signal is reduced by a factor $\Delta f T<1$. The complex envelope of the transmitted FDM symbol is given by

$$
s(t)=\frac{1}{\sqrt{T}} \sum_{n=0}^{N-1} S_{n} e^{j 2 \pi n \Delta f t}, \quad t \in[0, T],
$$

where $S_{n}$ is the modulation symbol for the $n^{\text {th }}$ carrier.

For channels impaired by Additive White Gaussian Noise (AWGN), $n(t)$, we can write the received signal $r(t)$ as

$$
r(t)=s(t)+n(t)
$$

The proposed FDM receiver consists of two stages. Initially, the received signal is processed by a bank of $N$ correlators that extract a set of sufficient statistics. The correlation processes are performed with a set of orthonormal basis functions $b_{n}(t)$, generated from the FDM carriers using the Gram Schmidt (GS) orthonormalisation method. Hence, the output of the $k^{\text {th }}$ receiver correlator can be expressed as

$$
R_{k}=\int_{0}^{T} r(t) b_{k}^{*}(t) d t, \quad k=0, \ldots N-1
$$




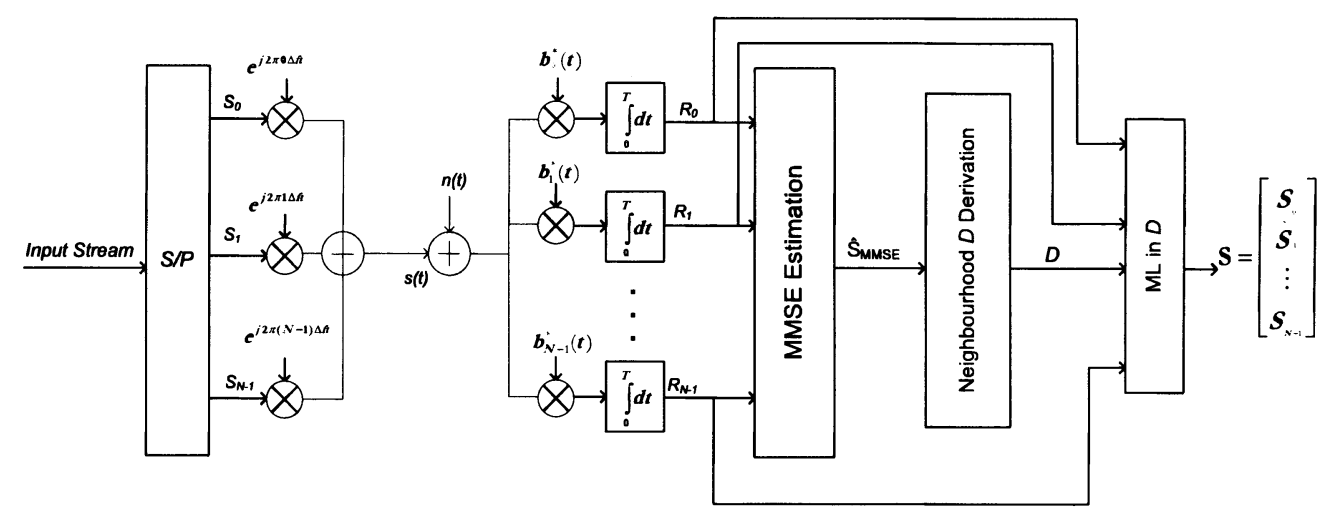

Fig. 1. The combined MMSE-ML GS FDM system architecture.

It is appropriate to describe the process by a linear statistical model

$$
\mathbf{R}=\mathbf{M S}+\mathbf{N},
$$

where $\mathbf{R}$ is the $N \times 1$ vector matrix of sufficient statistics, $\mathbf{S}$ is the $N \times 1$ vector of transmitted symbols, $\mathbf{M}$ is the $N \times N$ matrix of the correlation coefficients between the FDM carriers and the orthonormal base, and $\mathbf{N}$ is a vector of $N$ independent Gaussian noise samples with zero mean and covariance matrix $\sigma^{2} \mathbf{I}_{\mathbf{N}}\left(\mathbf{I}_{\mathbf{N}}\right.$ is the $N \times N$ identity matrix) [8]. Each of the elements of matrix $\mathbf{M}$ is given by

$$
m_{\alpha \beta}=\int_{0}^{T} e^{j 2 \pi(\alpha-1) \Delta f t} b_{(\beta-1)}^{*}(t) d t, \quad \alpha, \beta=1, \ldots, N
$$

The cross correlations matrix $\mathbf{M}$ is upper triangular [4], [9]; effectively, the output of each correlator is contaminated by intercarrier interference. Optimal detection (in terms of error performance) is based on the maximum likelihood principle. The ML estimate can be expressed as follows [4], [8]

$$
\hat{\mathbf{S}}_{\mathbf{M L}}=\underbrace{\arg \min }_{\mathbf{S}}\left\{\|(\mathbf{R}-\mathbf{M S})\|^{2}\right\}
$$

where $\|\cdot\|$ denotes the euclidean norm.

It is important to note that ML detection is impractical, because its computational complexity increases exponentially with the number of FDM carriers and the constellation cardinality. Therefore, we investigate the efficiency of two alternatives: i) linear MMSE detection and ii) linear MMSE detection combined with ML detection (see Fig. 1).

\section{FDM SIgNal Detection TeChNIQUeS}

\section{A. MMSE Detection}

In linear MMSE detection, the objective is to minimise the mean square error between the vector of transmitted symbols and the vector of their estimates, using solely linear operations represented by the matrix $\mathbf{G}_{\text {MMSE, }}$ i.e.,

$$
\begin{aligned}
\mathbf{G}_{\mathbf{M M S E}} & =\underbrace{\arg \min }_{\mathbf{G}} E\left\{\|\mathbf{S}-\hat{\mathbf{S}}\|^{\mathbf{2}}\right\} \\
& =\underbrace{\arg \min }_{\mathbf{G}} E\left\{\|\mathbf{S}-\mathbf{G R}\|^{\mathbf{2}}\right\}
\end{aligned}
$$

where $E\{\cdot\}$ is the expectation operator and $\mathbf{M}^{\mathrm{H}}$ denotes the hermitian of matrix $\mathbf{M}$.

It is straightforward to show that the MMSE matrix is

$$
\mathbf{G}_{\mathbf{M M S E}}=\mathbf{M}^{\mathrm{H}}\left(\mathbf{M} \mathbf{M}^{\mathrm{H}}+\frac{\sigma^{2}}{\sigma_{s}^{2}} \mathbf{I}_{\mathbf{N}}\right)^{-1}
$$

where $\sigma^{2}$ is the noise power and $\sigma_{s}^{2}$ is the power of the FDM signal (assuming transmitted symbols with $\sigma_{s}^{2} \mathbf{I}_{\mathbf{N}}$ covariance matrix.

Therefore, the MMSE estimate $\hat{S}_{M M S E}$ is

$$
\hat{\mathbf{S}}_{\mathbf{M M S E}}=\mathbf{M}^{\mathrm{H}}\left(\mathbf{M} \mathbf{M}^{\mathrm{H}}+\frac{\sigma^{2}}{\sigma_{s}^{2}} \mathbf{I}_{\mathbf{N}}\right)^{-1} \mathbf{R}
$$

We obtain the transmitted symbols estimates after slicing $\hat{\mathbf{S}}_{\text {MMSE }}$

\section{B. MMSE-ML Detection}

In MMSE-ML procedure, we use a two step procedure. Initially, we use an MMSE filter to generate an MMSE estimate $\hat{\mathbf{S}}_{\text {MMSE }}$ of the original transmitted symbols $\mathbf{S}$. Subsequently, we use the ML principle in a neighborhood, $\mathcal{D}$, of a sliced version of MMSE estimate. The neighborhood consists of the set of transmitted symbols whose binary representation is within a certain Hamming distance, $P$, from the binary representation (sliced version) of the MMSE estimate. This procedure is commonly known as boxed ML.

The neighborhood $\mathcal{D}$ consists of the set of transmitted vectors $\mathbf{S}$ obeying the relationship: $d_{H}\left(\mathbf{S}^{\prime}, \hat{\mathbf{S}}_{\text {MMSE }}^{\prime}\right) \leq P$ where $d_{H}(\cdot, \cdot)$ represents the Hamming distance operator, $\mathbf{S}^{\prime}$ represents the binary version of $\mathbf{S}$, and $\hat{\mathbf{S}}_{\mathbf{M M S E}}^{\prime}$ represents the binary version of $\hat{\mathbf{S}}_{\mathbf{M M S E}}$, i.e.,

$$
\mathbf{S} \in \mathcal{D} \text { iff } d_{H}\left(\mathbf{S}^{\prime}, \hat{\mathbf{S}}_{\mathbf{M M S E}}^{\prime}\right) \leq P
$$

\section{MMSE-ML Complexity}

The complexity of the proposed method depends on the number of calculations of both the MMSE and ML components of the algorithm. The former has a complexity of polynomial order $O\left(N^{3}\right)$ over the number of carriers $N$. The computational cost of the latter depends on the size of the MMSE neighborhood $\mathcal{D}$, since this determines the number of 
the executed ML comparisons. The length of the expanded FDM symbols is equal to $N \times \log _{2} M$. Consequently, the size of the neighborhood $\mathcal{D}$ will be equal to the sum of all possible combinations of $N \times \log _{2} M$ bits with $k$ flipped bits taken at a time, where $k$ runs from 1 to $P$, i.e.

$$
\begin{aligned}
\operatorname{size}(D) & =\sum_{k=1}^{P}\left(\begin{array}{c}
N \log _{2} M \\
k
\end{array}\right) \\
& =\sum_{k=1}^{P} \frac{\left(N \log _{2} M\right) !}{\left(N \log _{2} M-k\right) ! k !}
\end{aligned}
$$

It is apparent that for $P$ equal to zero, the introduced scheme reduces to MMSE. For $P$ equal to unity, the number of necessary boxed ML comparisons is $N \log _{2} M$, as opposed to the $M^{N}$ comparisons required for the ML implementation over the entire group of FDM symbols. Table I provides the ratio $\alpha$ of the number of ML over MMSE-ML comparisons for various FDM signal dimensions.

TABLE I

RATIO $\alpha$ OF ML OVER THE MMSE-ML COMPARISONS

\begin{tabular}{|c||c|c|c|c|}
\hline & BPSK & BPSK & QPSK & QPSK \\
\hline Carriers & $\alpha, d_{H}=1$ & $\alpha, d_{H}=2$ & $\alpha, d_{H}=1$ & $\alpha, d_{H}=2$ \\
\hline 2 & 2 & 1.33 & 4 & 1.6 \\
\hline 4 & 4 & 1.60 & 32 & 7.11 \\
\hline 8 & 32 & 7.11 & 4096 & 481.88 \\
\hline 16 & 4096 & 481.88 & $>10^{10}$ & $>8 \times 10^{6}$ \\
\hline
\end{tabular}

\section{Modified Gram Schmidt}

It is clear from section III that the inversion of matrix $\mathbf{M}$ is a prerequisite of the MMSE application. However, $\mathbf{M}$ eigenvalues (and consequently its determinant) go to zero rapidly with the increase of the number of carriers. Part of the problem is due to the orthonormalisation procedure. Classic GS (CGS) [4], [8] generates new vectors by subtracting from the respective initial carrier its projections (onto the already orthonormalised carriers). Unfortunately, the projections operation introduces numerical errors that result in the process degradation and consequently the loss of orthogonality after a number of calculations. A significant improvement is the Modified Gram Schmidt (MGS) method. While mathematically equivalent to CGS, MGS iterative nature results in a superior computational performance [10]. The main difference from the classic GS is that while in CGS the algorithm calculates the $m^{t h}$ vector and leaves the remaining $m+1$ to $N$ vectors unaltered, in the modified version after the calculation of each vector the remaining vectors are also transformed.

A simple implementation of the MGS is the following

for $m$ from 1 to $N$

$$
\begin{aligned}
& b_{m}(t)=\frac{1}{\sqrt{\xi_{m}}}\left[g_{m}(t)-\sum_{i=1}^{m-1} \int_{0}^{T} g_{m}(t) b_{i}^{*}(t) d t b_{i}(t)\right] \\
& \text { for } n \text { from } m+1 \text { to } N
\end{aligned}
$$

$$
g_{n}(t)=\frac{1}{\sqrt{\xi_{n}}}\left[g_{n}(t)-\sum_{i=1}^{n-1} \int_{0}^{T} g_{n}(t) b_{i}^{*}(t) d t b_{i}(t)\right]
$$

end of $n$ loop

end $m$ loop

where $\xi_{m}$ is chosen so that the energy of the $m^{t h}$ orthonormalised carrier is 1 .

Simulation results show that the adaptation of the new orthonormalisation technique enables doubling the dimension of the FDM signal without forcing the $\mathbf{M}$ matrix determinant to approach zero rapidly. Nevertheless, matrix singularity remains a fundamental limitation in signal detection of FDM systems operating below the orthogonality limit.

\section{RESUlts}

In our modelling we considered transmission over AWGN only. We performed Bit Error Rate (BER) measurements of simulated systems for the proposed MMSE and MMSE-ML schemes for up to $N=48$ FDM carriers with minimum frequency separation equal to 0.3 of the inverse of the FDM symbol period, $\Delta f=\frac{0.3}{T}$, and a fixed value of Energy Per Bit to Noise Density Ratio $\left(E_{b} / N_{0}\right)$ equal to $5 \mathrm{~dB}$. Carriers were modulated either by real BPSK or complex QPSK symbols. In all simulations, ML and/or ZF detection curves are used as reference.

\section{A. Error Performance}

Figures 2 and 3 show BER versus the normalised carrier distance (a fraction of the inverse of the FDM symbol period, $d F T=\Delta f \times T)$. Simulations were performed for a small number $(N=4)$ of BPSK and QPSK FDM carriers. MMSEML measurements were taken with Hamming distance $d_{H}=1$ for BPSK carriers and $d_{H}=\{1,2\}$ for QPSK carriers.

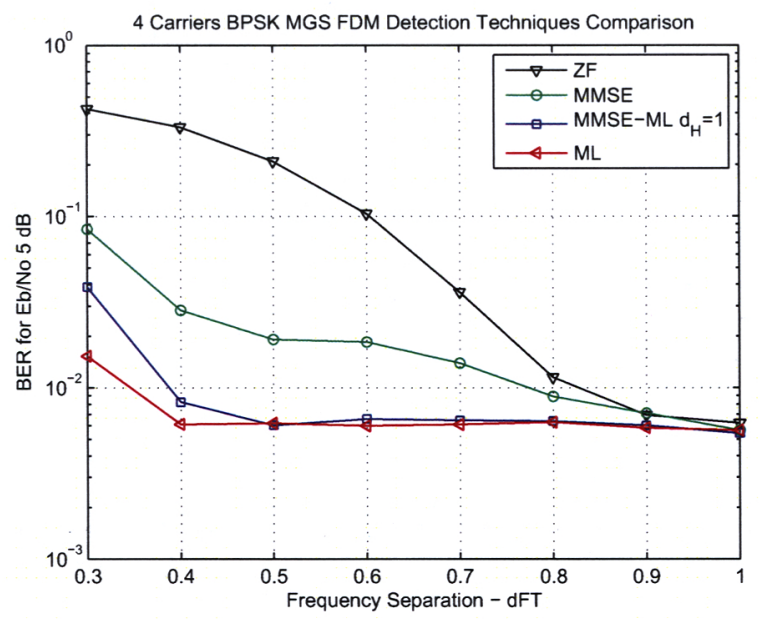

Fig. 2. BER of MMSE and MMSE-ML $\left(d_{H}=1\right)$ detection versus normalised frequency separation for $N=4$ BPSK FDM carriers. $E b / N_{0}$ is $5 \mathrm{~dB}$.

Both figures show that MMSE is superior to ZF but inferior to ML for BPSK and QPSK FDM signals. MMSE-ML 


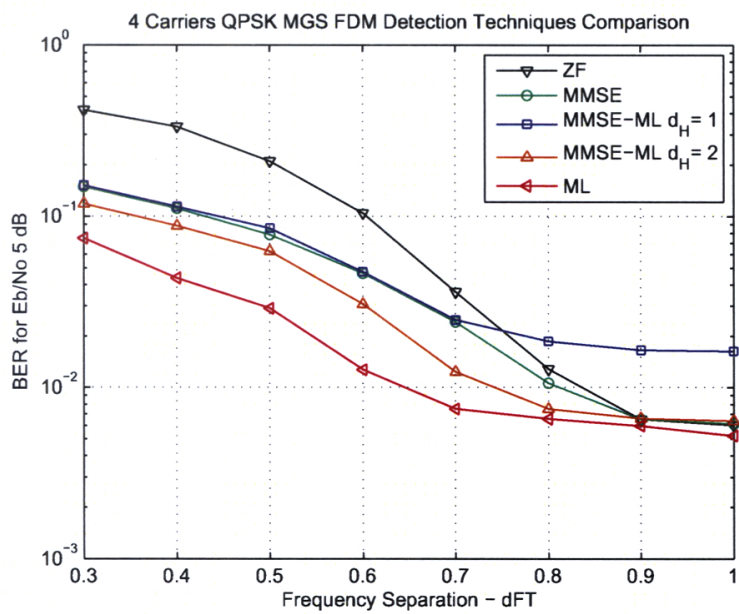

Fig. 3. BER of MMSE and MMSE-ML detection versus normalised frequency separation for $N=4$ QPSK FDM carriers. $E b / N_{0}$ is $5 \mathrm{~dB}$ and the Hamming distance in MMSE-ML scheme is $d_{H}=\{1,2\}$.

performs close to ML for BPSK FDM carriers. However, the BER in the QPSK case depends on the selected Hamming distance.

Further studies were performed for a larger number of BPSK carriers $N=4,8,24,36,48$ and an MMSE neighborhood with $d_{H}=1$. Fig. 4 shows that concatenating MMSEML detection introduces only a small error penalty as the number of BPSK FDM carriers increases.

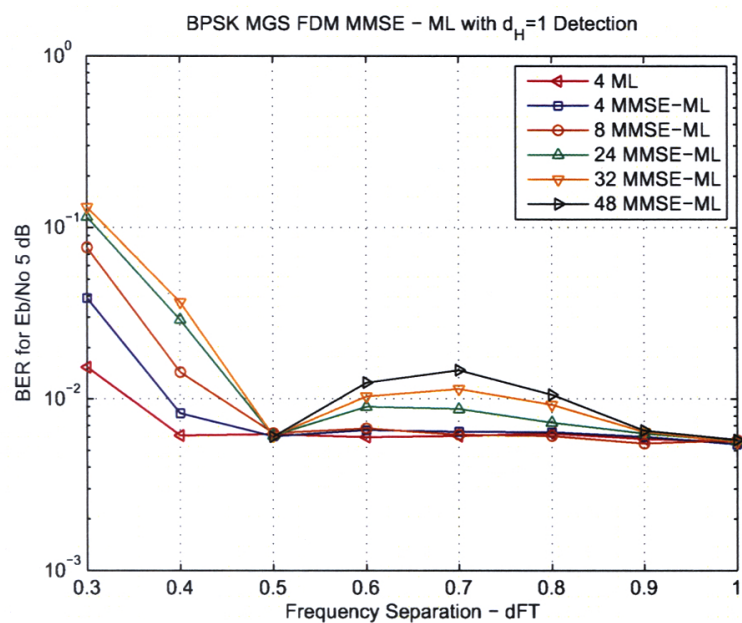

Fig. 4. BER of MMSE-ML $\left(d_{H}=1\right)$ detection versus normalised frequency separation for $N=\{4,8,24,32,48\}$ FDM carriers. $E b / N_{0}$ is $5 \mathrm{~dB}$.

In addition, we performed BER measurements for various $E b / N_{0}$ values, for a fixed $d F T=0.75$. Fig. 5 shows that BPSK performance almost matches the ML case. On the contrary, Fig. 6 shows that in the QPSK case the error performance depends on the length of the selected Hamming distance. For $d_{H}=1$, MMSE-ML performs worse than MMSE even for a small number of carriers. As the latter increases, a larger Hamming distance $\left(d_{H} \geq 2\right)$ is required to improve the single MMSE detection.

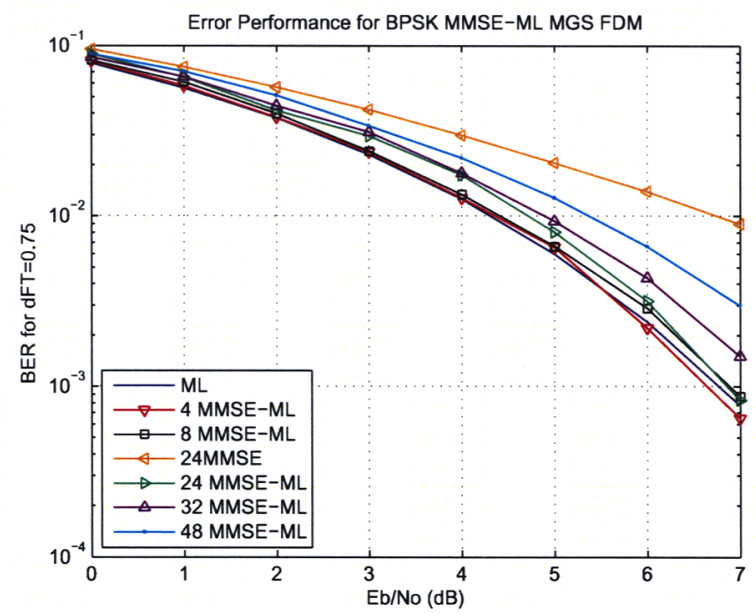

Fig. 5. BER of MMSE and MMSE-ML $\left(d_{H}=1\right)$ detection versus $E b / N_{0}$ for $N=\{4,8,24,36,48\}$ BPSK FDM carriers. The carriers distance is $d F T=\frac{0.75}{T}$.

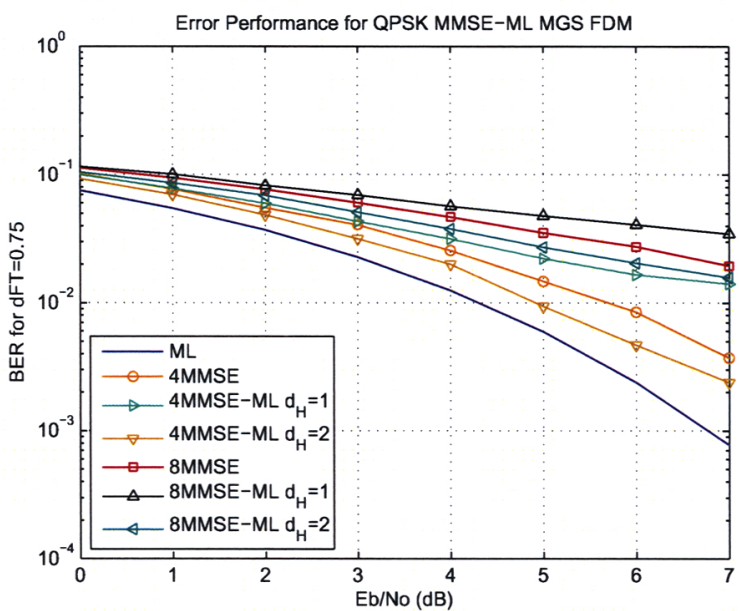

Fig. 6. BER of MMSE and MMSE-ML detection versus $E b / N_{0}$ for $N=$ $\{2,4,8,16\}$ QPSK FDM carriers. The carriers distance is $d F T=\frac{0.75}{T}$ and the Hamming distance in MMSE-ML scheme is $d_{H}=\{1,2\}$.

\section{B. Computational Complexity}

To estimate the computational complexity of the proposed methods, we measured the CPU execution time for different simulations scenarios. In all measurements, we considered the detection of $10 \mathrm{FDM}$ symbols for $E b / N_{0}=5 \mathrm{~dB}$ and $d F T=0.75$. In addition, we evaluated MMSE-ML for Hamming distances $d_{H}=\{1,2,3\}$.

Figures 7 and 8 illustrate a comparison of indicative CPU times between ZF, MMSE and MMSE - ML detection. It is 
clear that $\mathrm{ZF}$ and MMSE require less computational effort. MMSE - ML with $d_{H}$ equal to unity has simulation time comparable to that of MMSE. However, the order of its complexity increases with the size of the MMSE neighborhood.

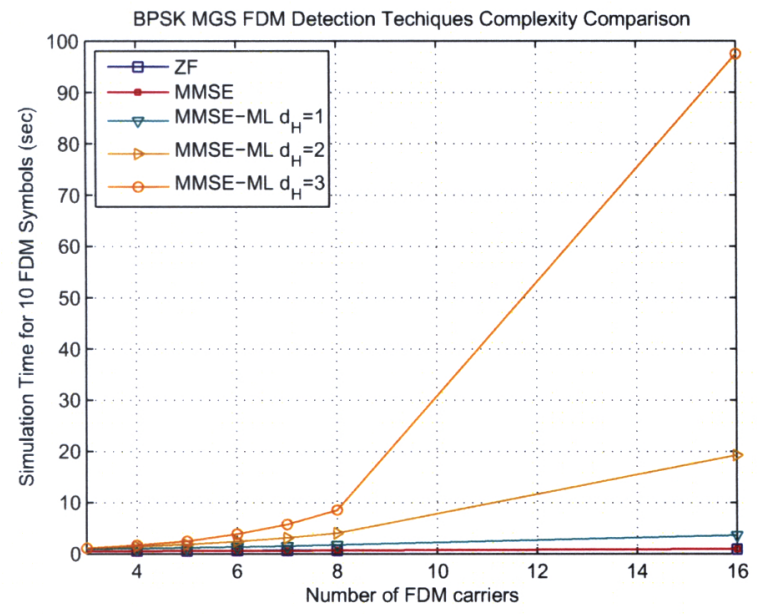

Fig. 7. CPU execution times for ZF, MMSE, and MMSE-ML FDM detection for 10 BPSK FDM symbols of $N=2$ to 16 carriers. The carriers distance is $d F T=0.75$ of the inverse of the FDM symbol period $T$. MMSE-ML detection is simulated for Hamming distance $d_{H}=\{1,2,3\}$.

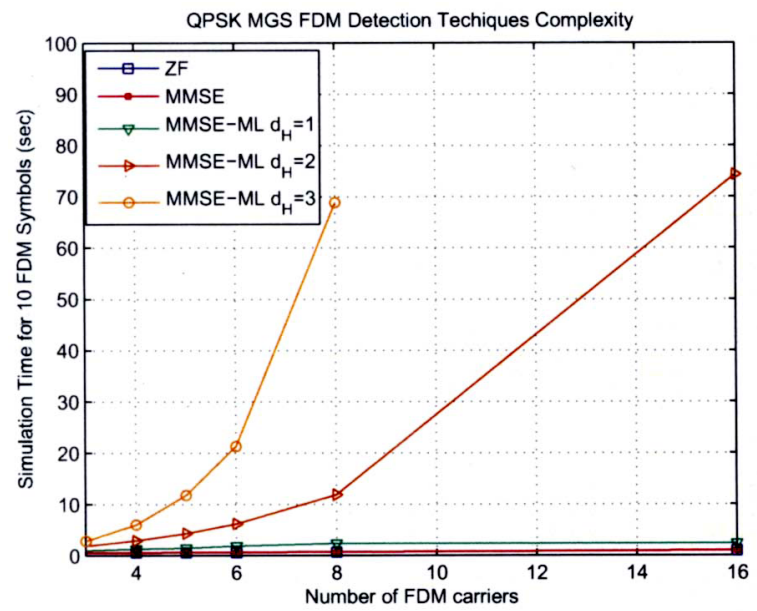

Fig. 8. CPU execution times for ZF, MMSE, and MMSE-ML FDM detection for 10 QPSK FDM symbols of $N=2$ to 16 carriers. The carriers distance is $d F T=0.75$ of the inverse of the FDM symbol period $T$. MMSE-ML detection is simulated for Hamming distance $d_{H}=\{1,2,3\}$.

\section{CONClusions And Future Work}

A new detection method for spectrally efficient FDM systems has been proposed. The method is based on combining
MMSE and ML. This results in a much reduced complexity and might lead to feasible implementation. Standard MMSE detection offers polynomial complexity at the expense of sub optimum error performance. To improve the latter, we applied ML in the neighborhood of the MMSE estimate. The order of the MMSE-ML combinations was derived and simulations were performed to evaluate its performance in AWGN. This new scheme performance is close to that of optimum ML for BPSK schemes at the expense of a small increase in the complexity. However, for QPSK the detection requires a larger number of comparisons that increase significantly with the number of the carriers.

In addition, the detectability of the signal is restricted by the signal dimension and the frequency separation that render the inversion of the cross correlations matrix impossible. Ongoing work includes investigating detection techniques such as Sphere Decoding [11], a mathematical algorithm that promises ML performance with reduced complexity for complex FDM signals.

\section{ACKNOWLEDGMENT}

This work is supported by the UK Engineering and Physical Sciences Research Council (EPSRC) under grant EP/D077362/1.

\section{REFERENCES}

[1] M. Rodrigues and I. Darwazeh, "Fast OFDM: A proposal for doubling the data rate of OFDM schemes," in Proceedings of the International Conference on Telecommunications, Beijing, China, Jun. 2002, pp. 484 487.

[2] F. Xiong, "M-ary amplitude shift keying OFDM system," IEEE Transactions on Communications, vol. 51, no. 10, pp. 1638-1642, Oct. 2003.

[3] D. Karampatsis, M. Rodrigues, and I. Darwazeh, "Performance comparison of OFDM and FOFDM communication systems in typical multipath environments: Effects of frequency selective fading, frequency and timing offsets and I/Q QAM modulator/demodulator imbalance," in 7th World Multiconference on Systemics, Cybernetics, and Informatics (SCI 2003), Orlando, USA, July 2003

[4] M. Rodrigues and I. Darwazeh, "A spectrally efficient frequency division multiplexing based communication system," in 8th International OFDMWorkshop, Hamburg, Germany, Sep. 2003, pp. 70-74.

[5] A. J. Paulraj, D. A. Gore, R. U. Nabar, and H. Blcskei, "An overview of MIMO communications A key to gigabit wireless," IEEE Proceedings, vol. 92, no. 2, pp. 198-218, February 2004.

[6] V. Pammer, Y. Delignon, W. Sawaya, and D. Boulinguez, "A low complexity suboptimal MIMO receiver: The combined ZF-MLD algorithm," 14th IEEE Proceedings on Personal, Indoor and Mobile Radio Communications, 2003. PIMRC 2003, vol. 3, pp. 2271-2275 vol.3, 7-10 Sept. 2003

[7] F. Wang, Y. Xiong, and X. Yang, "Approximate ML detection based on MMSE for MIMO systems," PIERS Online, vol. 3, no. 4, pp. 475-480, 2007.

[8] J. G. Proakis, Digital Communications 4th Edition. McGraw Hill, 2001

[9] L. L. Scharf, Statistical Signal Processing. Addison-Wesley, 1991.

[10] J. W. D. Ben Noble, Applied Linear Algebra, 2nd ed. Prentice Hall, 1977.

[11] E. Viterbo and J. Boutros, "A universal lattice code decoder for fading channels," IEEE Transactions on Information Theory, vol. 45, no. 5, pp. 1639-1642, Jul 1999. 